\title{
Complement Deposition and IgG Binding on Stored Red Blood Cells Are Independent of Storage Time
}

\author{
Astrid J.F. Thielen Elisabeth M. Meulenbroek Inge Baas Robin Bruggen \\ Sacha S. Zeerleder Diana Wouters
}

Department of Immunopathology, Sanquin Research, Amsterdam, the Netherlands

\section{Keywords}

Red blood cells - Storage - Complement activation . Antibody binding $\cdot$ Phagocytosis

\section{Summary}

Background: In the Netherlands, red blood cells (RBCs) are allowed to be stored up to 35 days at $2-6{ }^{\circ} \mathrm{C}$ in saline-adenine-glucose-mannitol (SAGM). During storage, RBCs undergo several changes that are collectively known as storage lesion. We investigated to what extent complement deposition and antibody binding occurred during RBC storage and investigated phagocytic uptake in vitro. Methods: RBCs were stored for different lengths of time at $2-6{ }^{\circ} \mathrm{C}$ in SAGM. Complement deposition and antibody binding were assessed upon storage and after incubation with serum. M1- and M2-type macrophages were generated from blood monocytes to investigate RBC phagocytosis. Results: No complement deposition was directly observed on stored RBCs, while incubation of RBCs with serum resulted in variable donor-dependent C3 deposition and IgG binding, both independent of storage time. Only $1-4 \%$ phagocytosis of stored RBCs by macrophages was observed. Conclusion: RBCs are susceptible to complement deposition and antibody binding independent of storage time. Limited phagocytic uptake by macrophages was observed in vitro.

(C) 2018 The Author(s)

Published by S. Karger GmbH, Freiburg

\section{Introduction}

Worldwide about 85 million units of red blood cells (RBCs) are transfused per year. Transfusion remains an essential therapy in the treatment of critically ill patients. However, $10-25 \%$ of donor RBCs are cleared from the circulation of transfused recipients within $24 \mathrm{~h}$ [1]. In the Netherlands, RBC concentrates (RCCs) may be stored up to 35 days at $2-6{ }^{\circ} \mathrm{C}$ in saline-adenine-guanine-mannitol (SAGM) before transfusion, which is more conservative than in most countries [2]. During storage, RBCs undergo several biochemical and structural changes, collectively known as 'storage lesion' $[3,4]$. Mostly retrospective and observational studies indicate that longer stored RBCs potentiate adverse clinical outcomes in transfusion recipients $[5,6]$. However, more recent multicenter randomized prospective studies did not confirm adverse outcomes such as in-hospital mortality [7-10]. To potentially improve in vivo survival of transfused RBCs, it is important to understand the mechanisms and consequences of the rapid clearance of transfused RBCs.

In vivo RBCs have a life span of $\sim 120$ days. At the end of their lifespan, RBCs become senescent, have decreased deformability, and are eventually phagocytosed by macrophages of the reticuloendothelial system [11]. Phagocytosis of senescent RBCs may be mediated by naturally occurring antibodies against band 3 and subsequent complement activation, exposure of phosphatidylserine (PS), and/or the molecular switch of CD47 that controls RBC phagocytosis [12].

During storage, the membrane of RBCs is altered which may result in complement activation, e.g. due to PS exposure, decreased expression of complement regulators such as decay-accelerating factor (DAF/CD55), CD59, and complement receptor 1 (CR1/ CD35) $[13,14]$. Alternatively, complement deposition may be mediated by bystander deposition via contact with the filter used for leukoreduction and/or plastic from the bag [15]. Szymanski and

Dr. Diana Wouters

Department of Immunopatholog

Sanquin Research

Plesmanlaan 125, 1066 CX Amsterdam, the Netherland

d.wouters@sanquin.n 
colleagues [16] showed that C3 fragments accumulate on the RBC membrane during storage of whole blood and that RBC survival was negatively correlated with both duration of storage and the amount of C3 deposition. When whole blood is separated followed by leukocyte depletion by filtration and storage of RBCs in SAGM, C3, C4 and C5 are undetectable in the RCC [17]. However, it has been reported that, despite leukoreduction, $\mathrm{C} 3$ is found on $\mathrm{RBC}$ membranes and soluble MAC levels are increased during $\mathrm{RBC}$ storage $[13,18]$.

Here, we investigated to what extent antibody binding and complement activation occurred during RBC storage. Furthermore, by incubating stored RBCs with normal human serum (NHS), we studied whether opsonization with complement and/or antibodies may contribute to in vitro phagocytic uptake of RBCs by M1- and M2-type macrophages.

\section{Material and Methods}

\section{Antibodies}

Monoclonal antibody (mAb) anti-C3-19, anti-C4-10, and anti-DAF-1 are in-house generated mAbs [19]. Anti-C3-19 was DyLight 488- or DyLight 647-conjugated (Thermo Scientific, Rockland, IL, USA). Anti-C4-10 and antiDAF-1 were DyLight 647-conjugated (Thermo Scientific). Polyclonal antibody anti-IgG-FITC was from Sanquin Reagents (Amsterdam, The Netherlands) Anti-CR1-PE (To5) was from Santa Cruz (Dallas, TX, USA) and anti-CD59CF405M (VJ1/12.2) was from Abcam (Cambridge, UK).

\section{Serum Samples}

Normal human serum was drawn from 13 donors, after informed consent. Eight sera were pooled (NHS pool), of which an aliquot was heat-inactivated (HI-NHS) for $30 \mathrm{~min}$ at $56^{\circ} \mathrm{C}, 5$ sera were individually aliquoted. All aliquots were stored at $-80{ }^{\circ} \mathrm{C}$.

\section{Isolation and Storage of RBCs}

RBCs from RCCs, collected in a quadruple citrate phosphate dextrose (CPD)-SAGM top-and-bottom bag system (Composelect, Fresenius HemoCare, Emmer-Compascuum, the Netherlands), were used from the Dutch Sanquin Blood Supply Foundation after informed consent from the donors. Alternatively, RBCs were isolated from whole blood collected in sodium citrate tubes by centrifugation at $1,080 \times g$ for $10 \mathrm{~min}$. After removing plasma and peripheral blood mononuclear cells, RBCs were washed three times with PBS by centrifugation at $1,080 \times g$ for 10 min (Fresenius Kabi, Zeist, the Netherlands) and stored as a 50\% cell suspension in SAGM (Fresenius Kabi).

Both RCCs and RBCs from tubes were stored for a short (2-6 days) or long (35-40 days) time period at $2-6{ }^{\circ} \mathrm{C}$, and before use stored $\mathrm{RBCs}$ were washed with PBS to investigate the intrinsic capacity of the RBCs to bind antibodies and activate the complement system.

\section{RBC Incubation with Human Serum for Complement Deposition and} Antibody Binding

$0.16 \%$ RBCs in a final volume of $100 \mu$ were incubated with $50 \%$ NHS or HI-NHS (v/v) diluted in veronal buffer ( $3 \mathrm{mmol} / \mathrm{l}$ barbital, $1.8 \mathrm{mmol} / \mathrm{l}$ sodium barbital, $145 \mathrm{mmol} / \mathrm{l} \mathrm{NaCl}, \mathrm{pH} 7.4$ ) containing $0.05 \%$ gelatin (w/v), $10 \mathrm{mmol} / \mathrm{l}$ $\mathrm{CaCl}_{2}$, and $2 \mathrm{mmol} / \mathrm{l} \mathrm{MgCl}_{2}$ at $37{ }^{\circ} \mathrm{C}$ overnight. To detect antibody binding, RBCs were incubated for $1 \mathrm{~h}$ at room temperature. Allogenic NHS was used, unless indicated otherwise.
FACS Analysis of Complement Deposition, Antibody Binding, and Markers on $R B C s$

NHS exposed RBCs were washed with Hepes buffer containing $1 \%$ human serum albumin (v/v), $10 \mathrm{mmol} / \mathrm{l}$ glucose and $2 \mathrm{mmol} / \mathrm{l} \mathrm{CaCl}_{2}$ and stained with antibodies in the dark for $30 \mathrm{~min}$ at room temperature. Anti-C3-19, antiC4-10, and anti-DAF-1: $1 \mu \mathrm{g} / \mathrm{ml}$; anti-IgG 1:100 dilution; anti-CR1: 1:25 and anti-CD59: 1:12.5. RBCs were analyzed using fluorescence-activated cell sorting (FACS) analysis. Percentage positive RBCs was determined for complement deposition and antibody binding. Median fluorescence intensity was used for the expression of DAF, CR1, and CD59.

\section{Phagocytosis Assay}

A phagocytosis assay was performed as described before [20]. In short, M1- and M2-type macrophages were generated from human monocytes by stimulation for 9 days with 10 ng/ml GM-CSF (CellGenix, Freiburg i.Br., Germany) or $50 \mathrm{ng} / \mathrm{ml}$ M-CSF (eBioscience, Vienna, Austria). Subsequently, M1and M2-type macrophages were incubated with NHS-opsonized or untreated carboxyfluorescein succinimidyl ester (CFSE)-labeled RBCs. As positive control, RBCs were opsonized with anti-RhD antibody. RBCs were added to M1or M2-type macrophages for $2 \mathrm{~h}$ and $1 \mathrm{~h}$, respectively. After incubation, nonphagocytosed RBCs were removed, using an isotonic ammonium chloride lysis buffer. Uptake of CFSE-labelled RBCs by macrophages was analyzed by FACS.

In addition, the cells were stained with May-Grünwald eosin-methylene blue modified solution (Merck) for 5 min and subsequently with Giemsa solution (Merck) for $30 \mathrm{~min}$. Light micrographs were taken using a Axiovert Scope. A1 microscope (Zeiss, Oberkochen, Germany).

\section{Statistics}

Data were analyzed using GraphPad Prism (version 6; GraphPad Software, San Diego, CA, USA). To compare C3 deposition and IgG binding between short and long stored RBCs, a Wilcoxon test was performed. For the phagocytosis assay, a two-way Anova test and a post-test a Tukey multiple comparisons test was performed.

\section{Results}

\section{Incubation of RBCs with Normal Human Serum Leads to C3 Deposition}

To determine whether complement activation occurs on RBCs during isolation and storage, $\mathrm{C} 3$ and $\mathrm{C} 4$ deposition on $\mathrm{RBC}$ membranes were analyzed by flow cytometry. No C3 or C4 deposition was observed on the membranes of RBCs obtained from either RCCs or tubes that were stored for a short period or a long period (fig. 1A).

Next, we incubated RBCs with allogeneic NHS as complement source. Using ABO mismatch as model system, C3 and C4 deposition was observed on the RBC membrane (fig. 2A). Matched RBCs and NHS were used in all further experiments, to rule out complement activation due to $\mathrm{ABO}$ mismatches. Incubation of stored RBCs from either RCCs or tubes with NHS, resulted in C3 deposition on the membrane of a subpopulation of RBCs. No C4 deposition was observed (fig. 1B), indicating alternative complement pathway activation. When analyzing RBCs from 7 individual donors that were incubated with the same NHS pool, the percentage of C3-positive RBCs varied between 2 and 27\% for RCCs or 10 and $35 \%$ for tubes (fig. 1C). However, C3 deposition was not significantly different between RBCs that were stored for a short or a long 
Fig. 1. C3 deposition on RBCs upon incubation with normal human serum. A Representative FACS histograms are shown of $\mathrm{C} 3$ and $\mathrm{C} 4$ deposition measured directly on short (2-6 days) and long stored (35-40 days) RBCs. B Representative FACS histograms are shown of $\mathrm{C} 3$ and $\mathrm{C} 4$ deposition measured on short (2-6 days) and long stored (35-40 days) RBCs upon incubation with NHS. C Percentages of C3 positive RBCs upon incubation with NHS are shown that were short (2-6 days) or long (35-40 days) stored ( $\mathrm{n}=7$ RBC donors). The Wilcoxon test showed no significance. Grey: RBCs without fluorescently labelled antibody, black solid: HI-NHS, blue dashed: short storage, and red dotted: long storage.
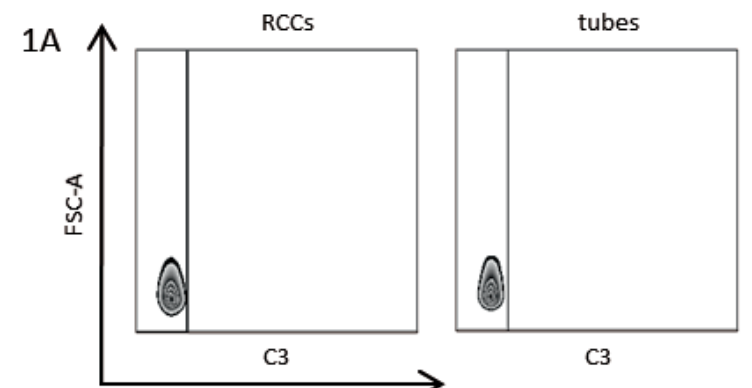

C3
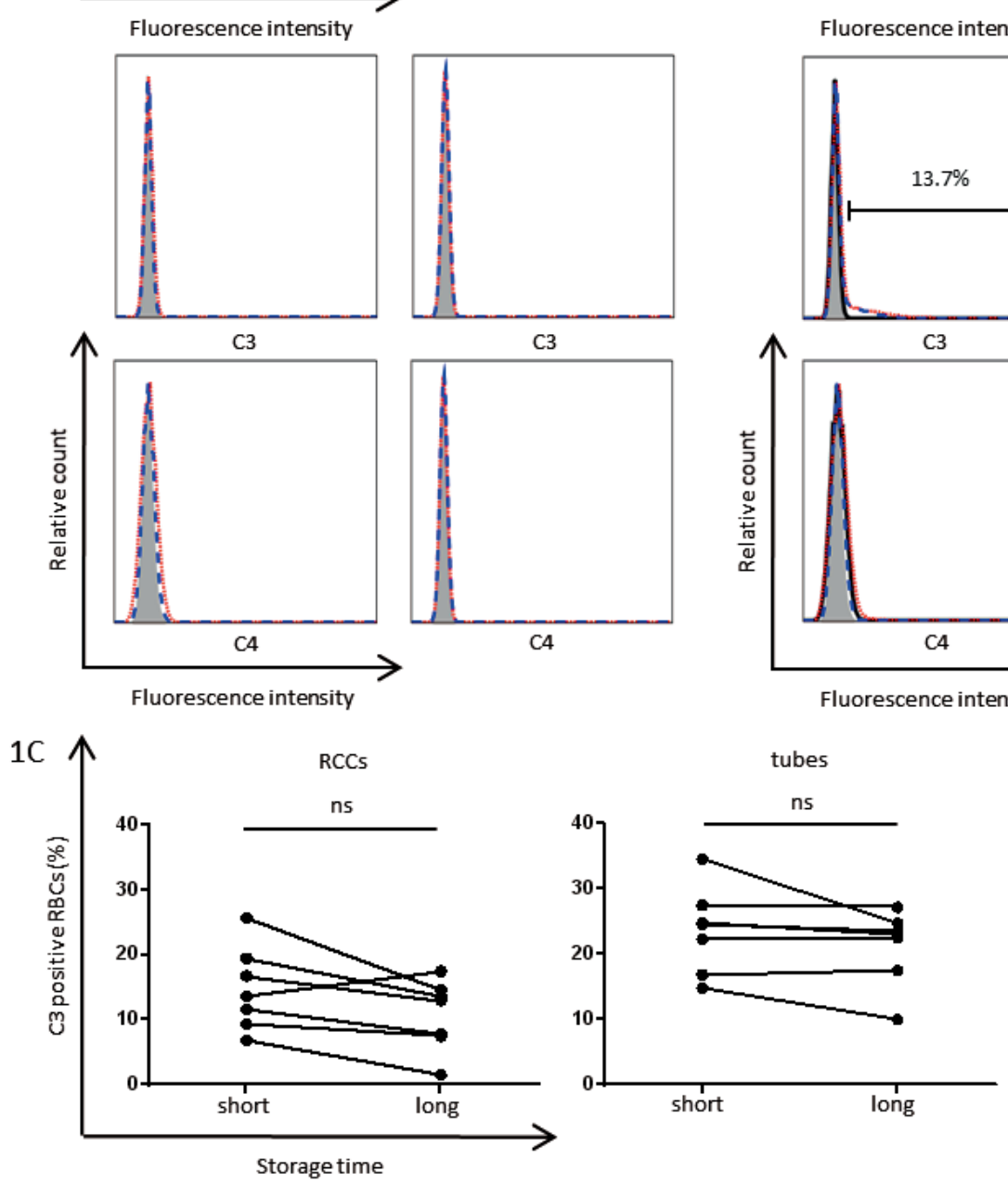
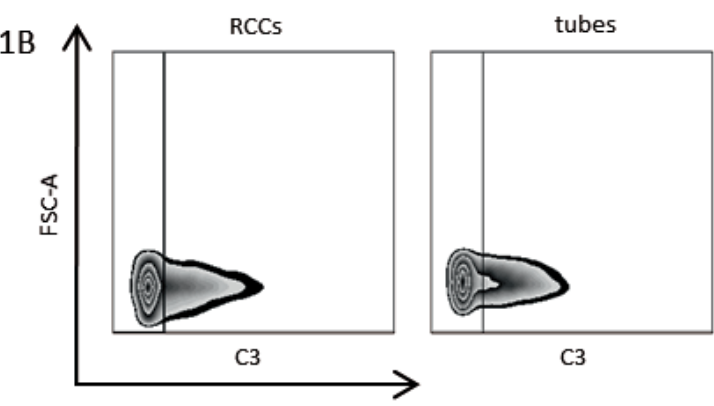

Fluorescence intensity

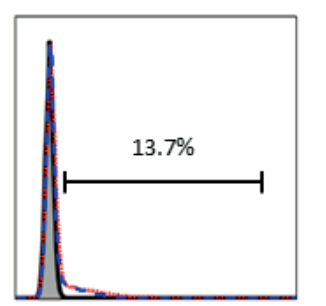

C3
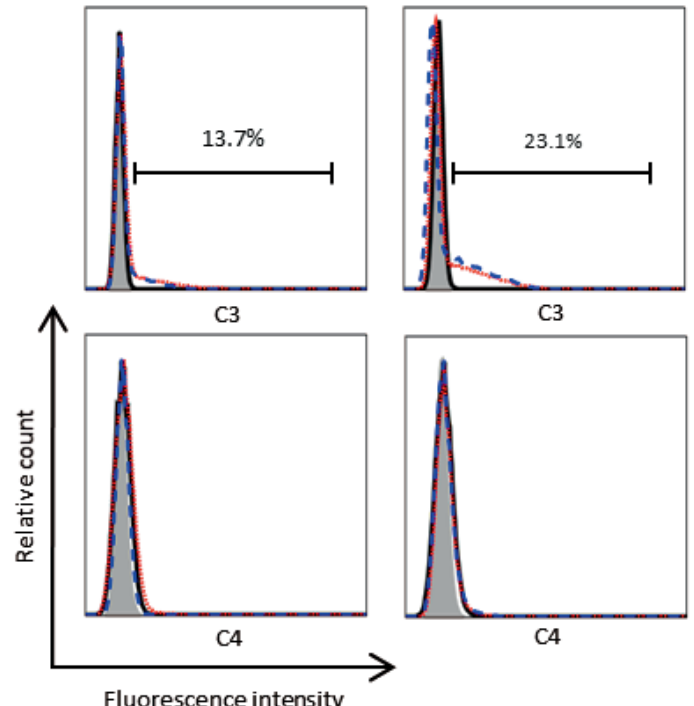

C3

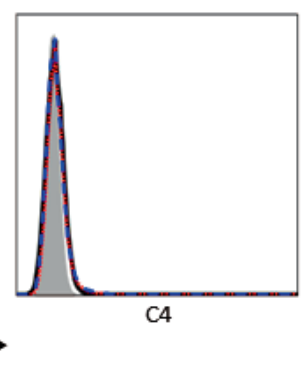

The total population of RBCs expressed DAF, CR1, or CD59

Table 1. DAF, CR1 and CD59 expression on short and long stored RBCs

\begin{tabular}{lcr}
\hline & \multicolumn{1}{l}{ Short } & \multicolumn{1}{c}{ Long } \\
\hline DAF & $780.6 \pm 125.7$ & $812.4 \pm 109.6$ \\
CR1 & $506.8 \pm 151.9$ & $502.8 \pm 151.0$ \\
CD59 & $9,012.8 \pm 1257.4$ & $8,925.8 \pm 903.4$ \\
\hline
\end{tabular}

Data shown as mean MFI $\pm \mathrm{SD}, \mathrm{n}=5$. No significant difference in Wilcoxon test between short (2-6 days) and long (35-40 days) stored RBCs.

period, indicating that $\mathrm{C} 3$ activation is $\mathrm{RBC}$ donor-dependent and independent of storage time. As no major differences were observed between RBCs from RCCs and tubes and to study the intrinsic capacity of the RBCs themselves, all further experiments were done with RBCs isolated from citrate tubes.

equally well on RBCs that were stored for a short or long period of time (table 1), and no correlation was found between expression of complement regulators and $\mathrm{C} 3$ deposition, excluding this as explanation for the observed C3-positive subset of RBCs.

\section{IgG Binds to Stored RBCs and Partly Coincides with Complement Activation}

Using ABO mismatch as model system, IgG binding was observed on the RBC membrane (fig. 2B). Matched RBCs and NHS were used in all further experiments to rule out antibody binding due to $\mathrm{ABO}$ mismatches. As shown in figure. 3A, IgG binding was detected on the RBCs after incubation with NHS. When analyzing RBCs from 5 individual donors incubated with the same NHS pool, the percentage of cells positive for IgG binding varied be- 
tween 10 and 21\%, and no significant difference was observed between RBCs that were stored for a short or a long period of time (fig. 3B)

Next, individual differences in IgG binding and C3 deposition were investigated by cross-incubating RBCs of 5 donors with serum of the same 5 donors (fig. $4 \mathrm{~A}$ ). Without serum incubation, no IgG binding or C3 deposition was observed on the RBCs (Q4). However, incubation of RBCs with allogeneic serum resulted in subsets of RBCs that were double-positive for both IgG and C3 (Q2) or single-positive for either C3 (Q1) or IgG only (Q3). The percentage of C3- and/or IgG-positive RBCs varied depending on the combination of RBCs and sera. Remarkably, even RBCs incubated with autologous serum showed C3 deposition and IgG binding (fig. 4B).

\section{$2 \mathrm{~A}$}

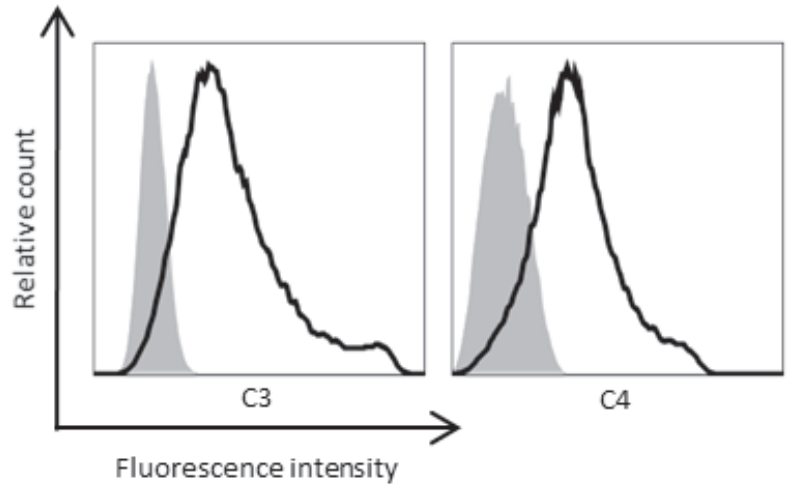

2B

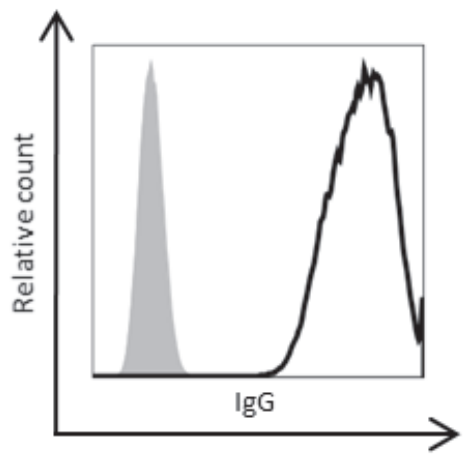

Fluorescence intensity

Fig. 2. Complement deposition and $\operatorname{IgG}$ binding in $A B O$ mismatch model. Representative FACS histograms are shown of $\mathbf{A} \mathrm{C} 3$ and $\mathrm{C} 4$ deposition and B IgG binding measured on blood group AB RBCs upon incubation with Otyped NHS. Grey: no NHS, black solid: NHS (representative graph of 3 individual experiments).
Phagocytic Uptake of RBCs by Macrophages Was Independent of Storage Time

Finally, we studied the phagocytic uptake of stored RBCs either opsonized with NHS or not. RBCs opsonized with anti-RhD antibody (positive control) were readily taken up, indicating that both in vitro generated allogeneic M1- and M2-type macrophages were capable of phagocytosing opsonized RBCs. M2-type macrophages phagocytosed the RhD-opsonized RBCs significantly more (64$80 \%)$ than M1-type macrophages (21-26\%), independent of storage time (fig. 5A). The extent of phagocytosis of both untreated RBCs and NHS-opsonized RBCs was low compared to RhD-opsonized RBCs, and no significant difference was observed between short and long stored RBCs. The percentage phagocytosis of both untreated RBCs and NHS-opsonized RBCs was lower for the M1type macrophages (average 1\%) compared to the M2-type macrophages (average $4 \%$ ) (fig. 5B).

\section{Discussion}

Complement deposition and/or antibody binding may contribute to rapid clearance of donor RBCs after blood transfusion. Here, we investigated whether complement activation and antibody binding occurred during RBC storage and whether this may contribute to phagocytic uptake in vitro.

No complement deposition or IgG binding was detected on stored RBCs from both RCCs and tubes stored at $2-6{ }^{\circ} \mathrm{C}$ in the standard storage solution SAGM. However, by incubating stored RBCs with allogeneic NHS, we observed C3 deposition on a subset of RBCs from both RCCs and tubes. RCC storage bags contain plasticizers, which are not present in the tubes, that can activate the complement system $[21,22]$. However, the percentage C3 deposition was somewhat lower on RBCs from the RCCs compared to those from the tubes, suggesting that under the investigated conditions plasticizers did not have a major effect on complement activation.

In contrast to a previously published study [13], we observed that storage time of RBCs had no influence on C3 deposition and IgG binding. In addition, we observed no difference in expression of complement regulators DAF (Cromer blood group system), CR1 (Knops blood group system), and CD59 over time, while Kamhieh-Milz et al. [13] suggested that increased complement
Fig. 3. IgG binding on RBCs upon incubation with normal human serum. A Representative FACS histograms are shown of IgG binding on short (2-6 days) and long (35-40 days) stored RBCs upon incubation with NHS. B Percentages of IgG positive RBCs upon incubation with NHS are shown that were short (2-6 days) or long (35-40 days) stored ( $\mathrm{n}=5 \mathrm{RBC}$ donors). The Wilcoxon test showed no significance. Grey: RBCs without fluorescently labelled antibody, blue dashed: short storage, and red dotted: long storage.
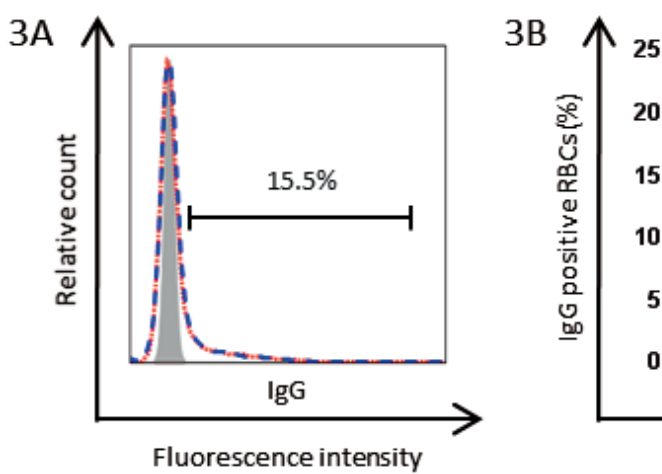

Storage time 
$4 \mathrm{~A}$

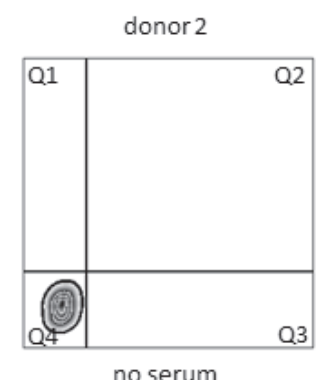

Fig. 4. C3 deposition and IgG binding partly coincides on RBCs upon incubation with normal human serum. A Representative FACS dot plots are shown of RBCs crossincubated with (autologous and allogeneic) serum of the same donors. Q1 = C3 deposition, Q2 = both C3 deposition and $\operatorname{IgG}$ binding, Q3 = IgG binding and $\mathrm{Q} 4=$ no C3 deposition and no IgG binding. B Percentages of IgG-positive RBCs (black), C3positive RBCs (white) and C3- and IgG-positive RBCs (grey) are shown ( $\mathrm{n}=5 \mathrm{RBC}$ donors and serum).
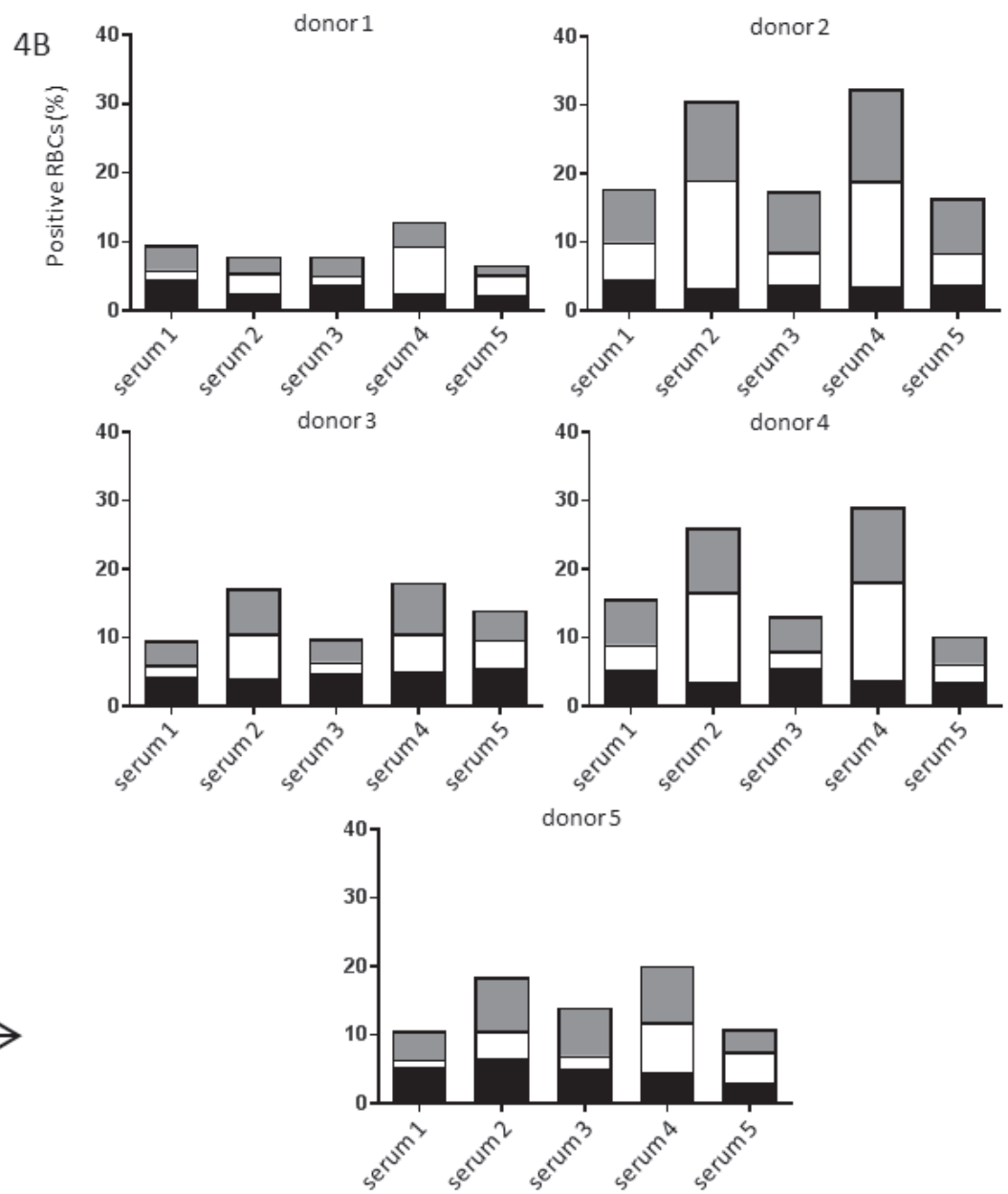

deposition during storage may be caused by diminished expression of complement regulators on RBCs. Kamhieh-Milz et al. [13] used phosphate-adenine-glucose-guanosine-saline-mannitol (PAGGS$\mathrm{M})$ as storage solution, while we used SAGM. It has been described that PAGGS-M prevents initial RBC swelling and spontaneous hemolysis, and osmotic fragility of the RBCs was lower compared to RBCs stored in SAGM [23]. However, it has not been investigated whether PAGGS-M influences complement activation, antibody binding, and the expression of complement regulators.

Interestingly, we observed a large donor variation for both $\mathrm{C} 3$ deposition and IgG binding on RBCs. For other factors such as hemolysis as well as osmotic and mechanical fragility also large donor variations are reported $[4,24]$. It has been described that genetic background and environmental factors may influence the properties of RBCs of individual blood donors, which make RBCs from some donors more prone to storage lesion than those of others [4, 24]. The amount of C3 and IgG opsonization may be an indication of the degree of storage lesion and therefore may act in the future as a biomarker for storage lesion. However, more studies are needed to investigate whether C3 and IgG opsonization on the subset of RBCs correlate with other known biomarkers of the storage lesion.

Most remarkably, even incubation with autologous serum resulted in both $\mathrm{C} 3$ deposition and IgG binding on RBCs. Changes are apparently induced in RBC membrane structures during blood collection and processing, which may lead to complement activation and antibody binding after transfusion.

Phagocytosis assays were performed to investigate whether the observed C3 deposition and IgG binding may contribute to clearance of stored RBCs. We observed that phagocytosis of RhD-opsonized RBCs by M1-type macrophages was significantly lower compared to that by M2-type macrophages. This is in agreement with other studies showing that monocytes activated with GM-CSF or interferon- $\gamma$ (M1-type macrophages), have a decreased phagocytic capacity compared to monocytes activated with M-CSF or IL-10 (M2-type macrophages) [20, 25]. Phagocytosis of untreated and NHS-opsonized RBCs was comparable, but low compared to $\mathrm{RhD}$-opsonized RBCs. Others have shown before that in case of allo- or autoantibodies, as occurring in a transfusion reaction or autoimmune hemolytic anemia, RBCs are cleared from the circulation by macrophages via $\mathrm{Fc}$ receptors and/or complement receptors $[26,27]$. It seems that the extent of C3 deposition and/or IgG binding on stored RBCs observed in our study was not sufficient for phagocytic uptake by macrophages.

In conclusion, no $\mathrm{C} 3$ deposition was observed on RBCs as present in a RCC, even upon prolonged storage. However, upon incubation of stored RBCs with serum, C3 deposition and IgG binding occur on a subset of RBCs. So likely, a fraction of donor RBCs will 
Fig. 5. $\operatorname{IgG}$ and $\mathrm{C} 3 \mathrm{op}-$ sonization on stored RBCs is not sufficient for phagocytic uptake by macrophages. A Wide field images and percentages phagocytosis are shown of the anti-RhD antibody (positive control) opsonized short (2-6 days) and long stored (35-40 days) RBCs $(\mathrm{n}=2)$ by M1- and M2-type macrophages. Two-way Anova test and as post-test a Tukey multiple comparisons test was performed and showed significance. B Percentages phagocytosis by M1- and M2type macrophages are shown of the untreated short (2-6 days) and long (35-40 days) stored RBCs $(\mathrm{n}=3)$ and NHSopsonized short (2-6 days) and long (35-40 days) stored RBCs ( $\mathrm{n}=$ 3). Bars show mean \pm standard deviation.
$5 A$
M1 macrophages

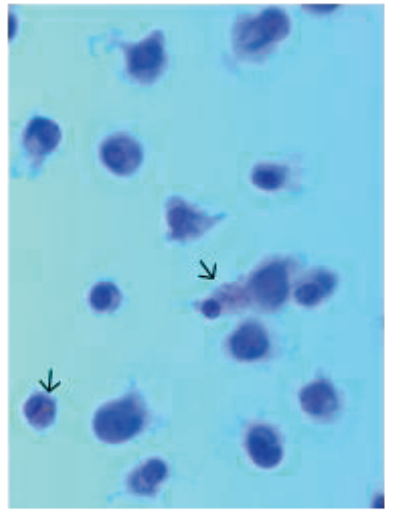

M2 macrophages

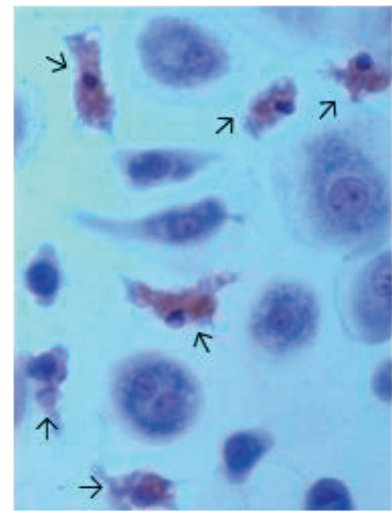

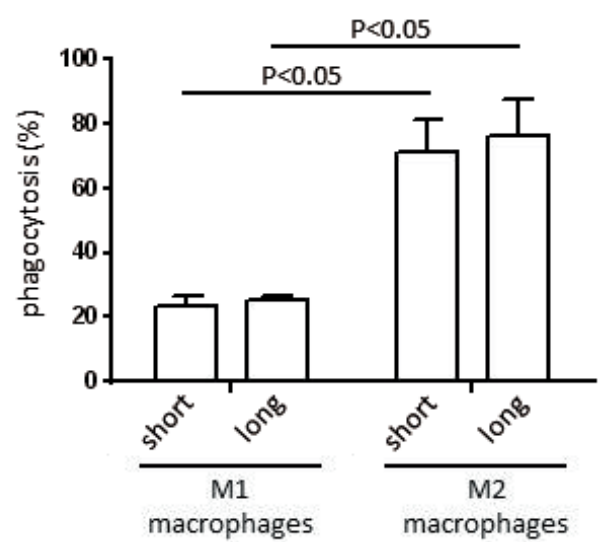

Anti-RhD
$5 B$

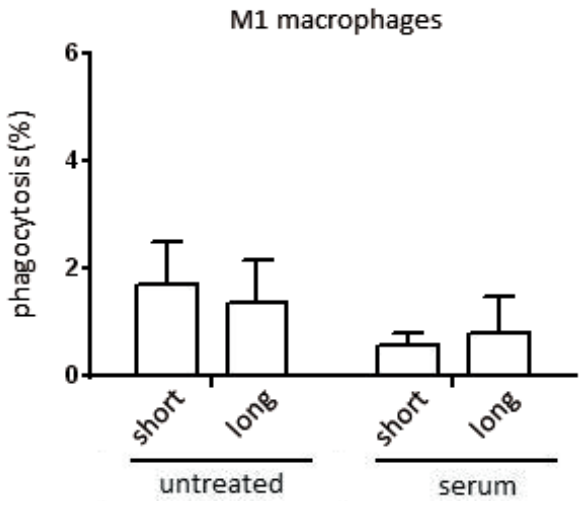

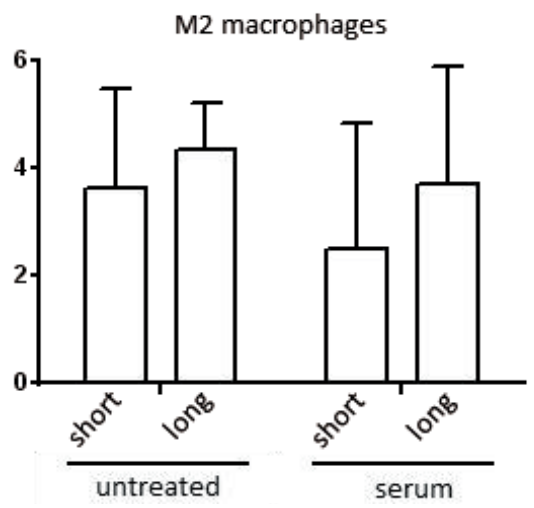

be opsonized with IgG and/or C3 after transfusion. This needs to be verified in vivo by tracing transfused RBCs in patients receiving a blood transfusion and measuring complement and $\operatorname{IgG}$ binding on the donor RBCs, which is subject of current research. Only minimal phagocytosis of RBCs was observed in vitro by allogeneic M1-and M2-type macrophages, which seemed to be independent of NHS opsonization. Apparently, phagocytosis by these cells was not significantly increased by C3 and IgG opsonization on the RBCs, although these cells do express complement receptors and Fc- receptors.

\section{Acknowledgments}

This work was supported by an internal grant of Sanquin (PPOC-12038-PRG)

\section{Disclosure Statement}

The authors declare that they have no competing interests.

\section{References}

1 Luten M, Roerdinkholder-Stoelwinder B, Schaap NP, de Grip WJ, Bos HJ, Bosman GJ: Survival of red blood cells after transfusion: a comparison between red cells concentrates of different storage periods. Transfusion 2008; $48: 1478-1485$.

2 Flegel WA, Natanson C, Klein HG: Does prolonged storage of red blood cells cause harm? Br J Haematol 2014;165:3-16.

3 van de Watering L: Red cell storage and prognosis. Vox Sang 2011;100:36-45.

4 Flatt JF, Bawazir WM, Bruce LJ: The involvement of cation leaks in the storage lesion of red blood cells. Front Physiol 2014;5:214

5 Purdy FR, Tweeddale MG, Merrick PM: Association of mortality with age of blood transfused in septic ICU patients. Can J Anaesth 1997;44:1256-1261.
6 Sanders J, Patel S, Cooper J, Berryman J, Farrar D, Mythen M, Montgomery HE: Red blood cell storage is associated with length of stay and renal complications after cardiac surgery. Transfusion 2011;51:2286-2294.

7 Lacroix J, Hebert PC, Fergusson DA, Tinmouth A, Cook DJ, Marshall JC, Clayton L, McIntyre L, Callum J, Turgeon AF, Blajchman MA, Walsh TS, Stanworth SJ, Campbell H, Capellier G, Tiberghien P, Bardiaux L, van de Watering L, van der Meer NJ, Sabri E, Vo D; ABLE Investigators; Canadian Critical Care Trials Group: Age of transfused blood in critically ill adults. N Engl J Med 2015;372:1410-1418.

8 Steiner ME, Ness PM, Assmann SF, Triulzi DJ, Sloan SR, Delaney M, Granger S, Bennett-Guerrero E, Blajchman MA, Scavo V, Carson JL, Levy JH, Whitman G, D'Andrea P, Pulkrabek S, Ortel TL, Bornikova
L, Raife T, Puca KE, Kaufman RM, Nuttall GA, Young PP, Youssef S, Engelman R, Greilich PE, Miles R, Josephson CD, Bracey A, Cooke R, McCullough J, Hunsaker R, Uhl L, McFarland JG, Park Y, Cushing MM, Klodell CT, Karanam R, Roberts PR, Dyke C, Hod EA, Stowell CP: Effects of red-cell storage duration on patients undergoing cardiac surgery. N Engl J Med 2015; 372:1419-1429.

9 Heddle NM, Cook RJ, Arnold DM, Liu Y, Barty R, Crowther MA, Devereaux PJ, Hirsh J, Warkentin TE, Webert KE, Roxby D, Sobieraj-Teague M, Kurz A, Sessler DI, Figueroa P, Ellis M, Eikelboom JW: Effect of short-term vs. long-term blood storage on mortality after transfusion. N Engl J Med 2017;375:1937-1945. 
10 Cook RJ, Heddle NM, Lee KA, Arnold DM, Crowther MA, Devereaux PJ, Ellis M, Figueroa P, Kurz A, Roxby D, Sessler DI, Sharon Y, Sobieraj-Teague M, Warkentin TE, Webert KE, Barty R, Liu Y, Eikelboom JW: Red blood cell storage and in-hospital mortality: a secondary analysis of the INFORM randomised controlled trial. Lancet Haematol 2017;4:e544-e552.

11 Mebius RE, Kraal G: Structure and function of the spleen. Nat Rev Immunol 2005;5:606-616.

12 de Back DZ, Kostova EB, van Kraaij M, van den Berg TK, van Bruggen R: Of macrophages and red blood cells; a complex love story. Front Physiol 2014;5:9.

13 Kamhieh-Milz J, Bartl B, Sterzer V, Kamhieh-Milz S, Salama A: Storage of RBCs results in an increased susceptibility for complement-mediated degradation. Transfus Med 2014;24:392-399.

14 Long KE, Yomtovian R, Kida M, Knez JJ, Medof ME: Time-dependent loss of surface complement regulatory activity during storage of donor blood. Transfusion 1993;33:294-300.

15 Seghatchian J: Universal leucodepletion: an overview of some unresolved issues and the highlights of lessons learned. Transfus Apher Sci 2003;29:105-117.

16 Szymanski IO, Odgren PR, Valeri CR: Relationship between the third component of human complement (C3) bound to stored preserved erythrocytes and their viability in vivo. Vox Sang 1985;49:34-41.
17 Hyllner M, Arnestad JP, Bengtson JP, Rydberg L, Bengtsson A: Complement activation during storage of whole blood, red cells, plasma, and buffy coat. Transfusion 1997;37:264-268.

18 Hu X, Patel RP, Weinberg JA, Marques MB, Ramos TN, Barnum SR: Membrane attack complex generation increases as a function of time in stored blood. Transfus Med 2014;24:114-116.

19 Meulenbroek EM, de Haas M, Brouwer C, Folman C, Zeerleder SS, Wouters D: Complement deposition in autoimmune hemolytic anemia is a footprint for difficult-to-detect IgM autoantibodies. Haematologica 2015;100:1407-1414.

20 Nagelkerke SQ, Dekkers G, Kustiawan I, van de Bovenkamp FS, Geissler J, Plomp R, Wuhrer M, Vidarsson G, Rispens T, van den Berg TK, Kuijpers TW: Inhibition of FcgammaR-mediated phagocytosis by IVIg is independent of IgG-Fc sialylation and FcgammaRIIb in human macrophages. Blood 2014;124: 3709-3718.

21 Lamba NM, Courtney JM, Gaylor JD, Lowe GD: In vitro investigation of the blood response to medical grade PVC and the effect of heparin on the blood response. Biomaterials 2000;21:89-96.
22 Zhong R, Wang H, Wu X, Cao Y, He Z, He Y, Liu J: In vitro investigation of the effect of plasticizers on the blood compatibility of medical grade plasticized poly (vinyl chloride). J Mater Sci Mater Med 2013;24:19851992

23 Zehnder L, Schulzki T, Goede JS, Hayes J, Reinhart WH: Erythrocyte storage in hypertonic (SAGM) or isotonic (PAGGSM) conservation medium: influence on cell properties. Vox Sang 2008;95:280-287.

24 Tzounakas VL, Georgatzakou HT, Kriebardis AG Voulgaridou AI, Stamoulis KE, Foudoulaki-Paparizos LE, Antonelou MH, Papassideri IS: Donor variation effect on red blood cell storage lesion: a multivariable, yet consistent, story. Transfusion 2016;56:1274-1286.

25 Capsoni F, Minonzio F, Ongari AM, Carbonelli V, Galli A, Zanussi C: IL-10 up-regulates human monocyte phagocytosis in the presence of IL- 4 and IFNgamma. J Leukoc Biol 1995;58:351-358.

26 Yazdanbakhsh K, Ware RE, Noizat-Pirenne F: Red blood cell alloimmunization in sickle cell disease pathophysiology, risk factors, and transfusion management. Blood 2012;120:528-537.

27 Berentsen S, Sundic T: Red blood cell destruction in autoimmune hemolytic anemia: role of complement and potential new targets for therapy. Biomed Res Int 2015;2015:363278 